\title{
AMENDMENTS
}

\section{Author Correction: NF-кB-inducing kinase maintains T cell metabolic fitness in antitumor immunity}

Meidi Gu, Xiaofei Zhou (D, Jee Hyung Sohn, Lele Zhu®i), Zuliang Jie, Jin-Young Yang, Xiaofeng Zheng, Xiaoping Xie (D, Jie Yang, Yaoyao Shi, Hans D. Brightbill, Jae Bum Kim (1), Jing Wang (D), Xuhong Cheng and Shao-Cong Sun (D)

Correction to: Nature Immunology https://doi.org/10.1038/s41590-020-00829-6, published online 4 January 2021.

In the version of this article initially published, affiliation 3 and the number for the grant awarded to J.B.K by the National Research Foundation, funded by the South Korean government, were incorrect. The errors have been corrected in the HTML and PDF versions of the article.

Published online: 11 February 2021

https://doi.org/10.1038/s41590-021-00892-7

() The Author(s), under exclusive licence to Springer Nature America, Inc. 2021

\section{Author Correction: $\mathrm{BACH} 2$ enforces the transcriptional and epigenetic programs of stem-like $\mathrm{CD}^{+} \mathrm{T}$ cells}

Chen Yao (1), Guohua Lou, Hong-Wei Sun (D), Ziang Zhu, Yi Sun, Zeyu Chen, Daniel Chauss, E. Ashley Moseman, Jun Cheng, Marc A. D’Antonio, Wangke Shi, Junwei Shi(D, Kohei Kometani, Tomohiro Kurosaki (D) E. John Wherry (D), Behdad Afzali, Luca Gattinoni (D), Yuwen Zhu, Dorian B. McGavern (D), John J. O'Shea, Pamela L. Schwartzberg and Tuoqi Wu(B)

Correction to: Nature Immunology https://doi.org/10.1038/s41590-021-00868-7, published online 11 February 2021.

In the version of this article initially published, the affiliation for author John J. O'Shea was given as Department of Surgery, Division of Surgical Oncology, University of Colorado Anschutz Medical Campus, Aurora, CO, USA. The correct affiliation is Molecular Immunology and Inflammation Branch, National Institute of Arthritis and Musculoskeletal and Skin Diseases, National Institutes of Health, Bethesda, MD, USA. The error has been corrected in the HTML and PDF versions of the article.

Published online: 3 March 2021

https://doi.org/10.1038/s41590-021-00906-4

(๑) The Author(s), under exclusive licence to Springer Nature America, Inc. 2021

\section{Publisher Correction: Fiber-poor Western diets fuel inflammation}

Robert T. Patry and Cathryn R. Nagler (D)

Correction to: Nature Immunology https://doi.org/10.1038/s41590-021-00880-x, published online 11 February 2021.

In the version of this article initially published, in Fig. 1, the red and green arrows in the bottom panel were switched. The green upward arrow should be next to TNF, IFN- $\gamma$ and IL-6, and the red downward arrow should be next to IL-10. The error has been corrected in the HTML and PDF versions of the article.

Published online: 4 March 2021

https://doi.org/10.1038/s41590-021-00907-3

(c) The Author(s), under exclusive licence to Springer Nature America, Inc. 2021 